Int. J. Dev. Biol. 55: 121-126 (2011)

doi: $10.1387 / \mathrm{ijdb} .103130 \mathrm{sn}$

\title{
The transcriptional coactivators Yap and TAZ are expressed during early Xenopus development
}

\author{
SUSUMU NEJIGANE ${ }^{1}$ Y YOSHIKAZU HARAMOTO ${ }^{2}$, MAKOTO OKUNO ${ }^{1}$, \\ SHUJI TAKAHASHI*,2 and MAKOTO ASASHIMA ${ }^{1,3}$ \\ ${ }^{1}$ Department of Life Sciences (Biology), ${ }^{2}$ Center for Structuring Life Sciences, Graduate School of Arts and \\ Sciences, The University of Tokyo and ${ }^{3}$ Research Center for Stem Cell Engineering, National Institute of \\ Advanced Industrial Sciences and Technology (AIST), Japan
}

\begin{abstract}
The Yap and TAZ genes encode highly conserved domains which bind various transcription factors. Yap and TAZ act as transcriptional coactivators to modulate transcriptional activity. The activities of Yap and TAZ are negatively regulated by Hippo signaling via direct phosphorylation. In this study, we describe the expression patterns of Yap and TAZ during the development of Xenopus tropicalis. The Xenopus tropicalis Yap (xtYap) and Xenopus tropicalis TAZ (xtTAZ) genes are expressed maternally. xtYap is widely expressed throughout embryogenesis, particularly in the facial connective tissues, branchial arch, midbrain-hindbrain boundary, otic vesicle, pronephros, notochord, hindgut and tailbud. xtTAZ expression occurs predominantly in the presomitic mesoderm, facial connective tissues, brain, branchial arch, trunk neural crest cells and migrating hypaxial myoblasts. In the muscle lineage, xtTAZ expression is transient and restricted to proliferating cells, the presomitic mesoderm and the edges of the hypaxial myoblasts, with no expression detected in mature muscle cells. These results provide insights into the functions of Yap and TAZ and their regulation by Hippo signaling during early development in Xenopus.
\end{abstract}

KEY WORDS: Yes-associated protein, Yap, TAZ, WWtr1, Xenopus

The Hippo pathway was originally identified in the fly as a controller of organ size, and its components are evolutionarily conserved in vertebrates (reviewed in Wang et al., 2009; Badouel et al., 2009). The core components of Hippo, Sav, Wts, and Mats in the fly are conserved in mammals as Mst1/2, WW45, LATS1/ 2 , and Mob1, respectively. Protein interaction screenings in Drosophila revealed that Hippo signaling negatively regulates Yorkie through direct phosphorylation. In the absence of Hippo signaling, Yorkie can promote transcription together with binding partners. The Yes-associated protein (Yap) and transcriptional coactivator with PDZ-binding motif (TAZ) are vertebrate homologs of Yorkie. Yap and TAZ show conservation of multiple functional domains, i.e., the TEF/TEAD binding domain, 14-3-3 binding motif, WW domain, and transactivation domain. In similarity to Yorkie, Yap and TAZ bind to transcription factors and act as transcriptional coactivators.

The overexpression of $Y a p$ and $T A Z$ induces cell proliferation, epithelial-mesenchymal transition, and anti-apoptosis (Overholzer et al., 2006; Lei et al., 2008). Gene ablation of Yap results in embryonic lethality, with defects in yolk sac vasculogenesis, chorioallantoic fusion, and embryonic axis elongation in the mouse (Morin-Kensicki et al., 2006). The injection into zebrafish of Yap-specific morpholino oligonucleotides results in the phenotype of small head with smaller eyes, no tail, and cardiac edema (Jiang et al., 2009). TAZ-knockout mice show partial lethality; survivors have renal cysts and slightly shorter skeletons (Hossain et al., 2007; Tian et al., 2007; Makita et al., 2008). TAZmorphants of zebrafish show cardiac edema, ventral curvature, and lack of ossification (Hong et al., 2005; Tian et al., 2007). These studies indicate that the functions of Yap and TAZ and their regulation by Hippo signaling are essential for the development of multiple tissues, and that Yap and TAZ play distinct roles in normal

Abbreviations used in this paper: orf, open reading frame; Yap, Yes-associated protein.

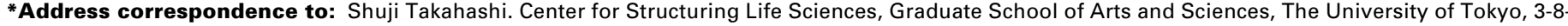
1 Komaba, Meguro-ku, Tokyo, Japan. Fax: +81-35-465-8838. e-mail: shujit@csls.c.u-tokyo.ac.jp
} 
vertebrate development.

Previous studies have demonstrated that Tead, Runx, Tbx5, Pax3, and other transcriptional factors are binding partners of Yap and TAZ (reviewed in Wang et al., 2009). These transcriptional factors are differentially expressed and function in several developmental processes. Yap and TAZ may also be required as transcriptional coactivators in each process. Elucidating the spatiotemporal expression patterns of $Y a p$ and $T A Z$ in vertebrate embryogenesis is important for understanding how Hippo signaling is involved in differentiation, tissue size control, and cell proliferation. In the present study, we reveal the expression patterns of Xenopus tropicalis Yap and TAZ genes, thereby providing insights into the functions of $Y$ ap and $T A Z$ in early development.

\section{Results}

\section{Molecular cloning of the xtYap and xtTAZ genes}

The sequences of the open reading frames (ORFs) of $x t Y a p$ and $x t T A Z$ were obtained from the genome databases. We performed PCR-based cloning using the cDNA species derived from stage 10.5 embryos and adult testes, respectively. The fulllength ORFs of $x t Y a p$ and $x t T A Z$ (GenBank accession numbers AB551789 for $x t Y a p$ and AB551790 for $x t T A Z$ ) encode 456 and 390 amino acids, respectively. The deduced amino acid sequences were aligned and compared with their homologs in other vertebrates (Fig. 1-3). The xtYap protein showed $76.4 \%, 78.0 \%$, and $75.4 \%$ identities with the human Yap (hYAP), mouse Yap (mYap), and zebrafish Yap (zYap), respectively. The xtTAZ protein showed $76.2 \%, 74.0 \%$, and $62.2 \%$ identities with hTAZ, mTAZ, and zTAZ, respectively. Although the functional domains of these proteins are conserved across species, a proline-rich motif that is conserved in the $\mathrm{N}$-terminal regions of the mYap and hYAP proteins is not present in the xtYap and zYap proteins.

\section{Expression patterns of xtYap and xtTAZ}

We performed semi-quantitative RT-PCR analyses of embryos and adult tissues (Fig. 4). During embryogenesis, $x t$ Yap transcripts were detected continuously from the unfertilized egg to the tadpole stages. The levels of $x t T A Z$ transcripts were low from the unfertilized egg to gastrula stages, but gradually increased from the neurula stage to tadpole stage (Fig. 4A). In the adult tissues,' $x$ tYap transcripts were found to be abundant in the gut, ovary and testis. In addition, xtYap was expressed at moderate levels in the heart and liver, whereas it was weakly expressed in the eyes and skin. The levels of $x t T A Z$ transcripts were high in the gut, heart, skin, and testes. XtTAZ was also expressed at moderate levels in the eyes, liver and ovaries, whereas it was weakly expressed in the muscles (Fig. 4B).

To examine the spatial expression patterns of these genes during the early development of Xenopus, we performed whole-mount in situ hybridiza-

Fig. 1. Comparisons of the Yap protein sequences of Xenopus tropicalis and other vertebrates. The scheme of xtYap is shown on the top. The amino acid sequence of xtYap was aligned with those of human Yap (hYAP, accession no. NP_001123617), mouse Yap (mYap NP_033560), and zebrafish Yap (zYap; NP_001132952).

The dark or light background highlights identical or similar

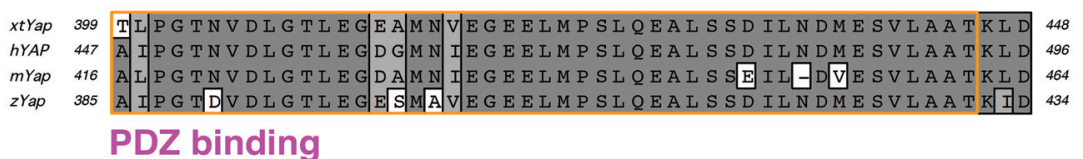

xtrap 449 KE S F T TWL 456

hYAP 497 K E S F L T W L 504

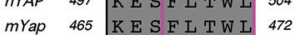

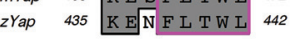

residues, respectively. The domains of Yap are boxed using the following colors and abbreviations; light-green, TB: Tead binding domain; blue, 14-3-3: 14-3-3 binding motif; red, WW: WW domain; light-blue, SH3: SH3 binding motif; yellow, TA: transcriptional activation domain; pink, PDZ: PDZ binding motif. 
tion analysis. The $x$ tYap transcripts were localized to the animal hemisphere until the early gastrula stage (Fig. 5 A-C). During gastrulation, the embryo was covered with xtYap-expressing ectodermal cells (Fig. 5D). At the neurula stage, $x t$ Yap was mainly expressed in the center of the neural plate and in the cranial neural crest cells (Fig. $5 \mathrm{E}, \mathrm{F}, \mathrm{J}$ ). From the tailbud stage to the tadpole stage, xtYap expression was evident in the facial connective tissues, branchial arch, midbrain-hindbrain boundary, otic vesicle, pronephros, notochord, hindgut (cloaca), and tailbud (Fig. 5 G-I). Histologic sectioning of a stage 30 embryo revealed that $x t Y a p$ was expressed in the ectodermal and mesodermal derivatives (Fig. 5K).

The expression of $x t T A Z$ was initially detected in the paraxial mesoderm at the late neurula stage (Fig. 6 $A, B, D)$. Thereafter, $x t T A Z$ expression was detected in the hindbrain and presomitic mesoderm from the early tailbud stage (Fig. $6 \mathrm{C}, \mathrm{E}, \mathrm{K}, \mathrm{L}$ ). It is noted that $\mathrm{xtTAZ}$ was not detected in the matured somite (Fig. $6 \mathrm{E}, \mathrm{J})$. In a transverse section of the tailbud stage, xtTAZexpression was observed in the heart, hindbrain, and the trunk neural crest cells (Fig. $6 \mathrm{H}-\mathrm{L}$ ). At the late tailbud stage, $x t T A Z$ expressed was detected in the facial connective tissues, midbrain-hindbrain boundary, hindbrain, branchial arch, and trunk neural crest cells. From embryonic stage 35, xtTAZ was expressed in the migrating hypaxial myoblasts, including the facial musculature (Fig. 6 $F, G, M, N)$.

\section{Discussion}

The Xenopus tropicalis Yap and TAZ proteins contain conserved domains, which include not only transcriptional factor binding sites, such as the Tead binding domain and WW domain, but also other functional domains (Fig. 1,2). However, a proline-rich region that is conserved in the $\mathrm{N}$-terminal regions of the chick, murine, and human Yap proteins is not present in the Xenopus or zebrafish Yap proteins. It has been reported that this proline-rich region interacts with the $\mathrm{SH} 3$ domain of cYes and other proteins (Sudol, 1994).

The expression patterns of Yap and TAZ during embryogenesis have been studied in mice and zebrafish. Yap transcripts have been detected at the 1-cell stage of mouse development (Nishioka et al., 2009). The murine Yap gene is expressed in almost all tissues, with the exceptions of the visceral and definitive endoderm. In particular,Ä $m Y a p$ is strongly expressed in the extraembryonic ectoderm and epiblast at E6.5. In addition to these regions, $m Y a p$ is expressed in the extraembryonic mesoderm and chorion at E7.5, and at the distal tip of the allantois at E8.5 (Morin-Kensicki et al., 2006). The $m Y a p$ expression patterns at later developmental stages have not been analyzed. In zebrafish, zYap transcripts exist maternally. Zygotic zYap is mainly expressed in the notochord, brain, eyes, branchial arches, and pectoral fins (Jiang et al., 2009). In the present study, we show that $x t Y a p$ is maternally expressed and is widely expressed throughout the developmental process, with strong expression in the center of neural plate and in the cranial neural crest cells at the neurula stage. We also demonstrate that $x t Y a p$ is strongly expressed in the facial connective tissues, branchial arch, midbrain-hindbrain boundary, otic vesicle, pronephros, notochord, hindgut, and tailbud at the tailbud stage.

$T A Z$ is expressed from the 1-cell stage of the mouse embryo (Nishioka et al., 2009). TAZ is localized to the forebrain, hindbrain, and somites between E9.0 and E10.5 of mouse embryogenesis (Di Palma et al., 2008). In the present study, we show that xtTAZ is maternally expressed and is expressed in the hindbrain and
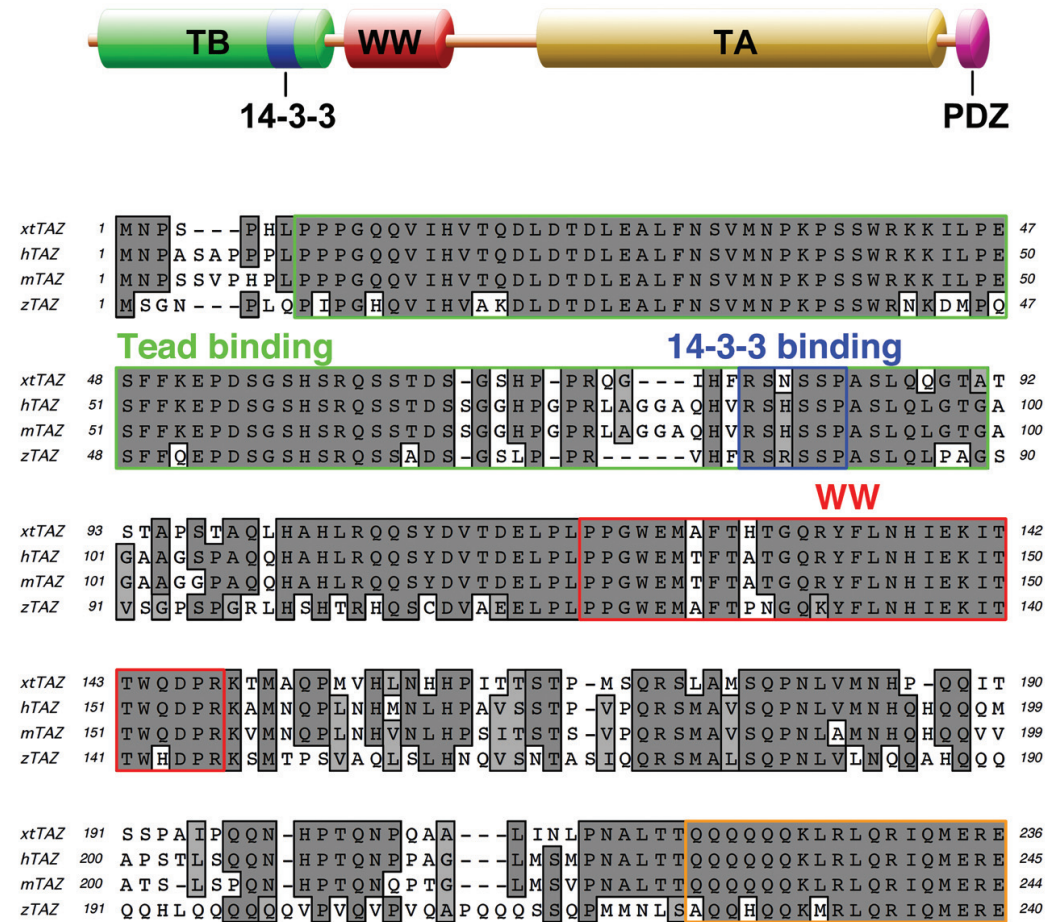

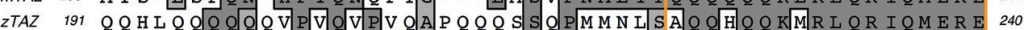
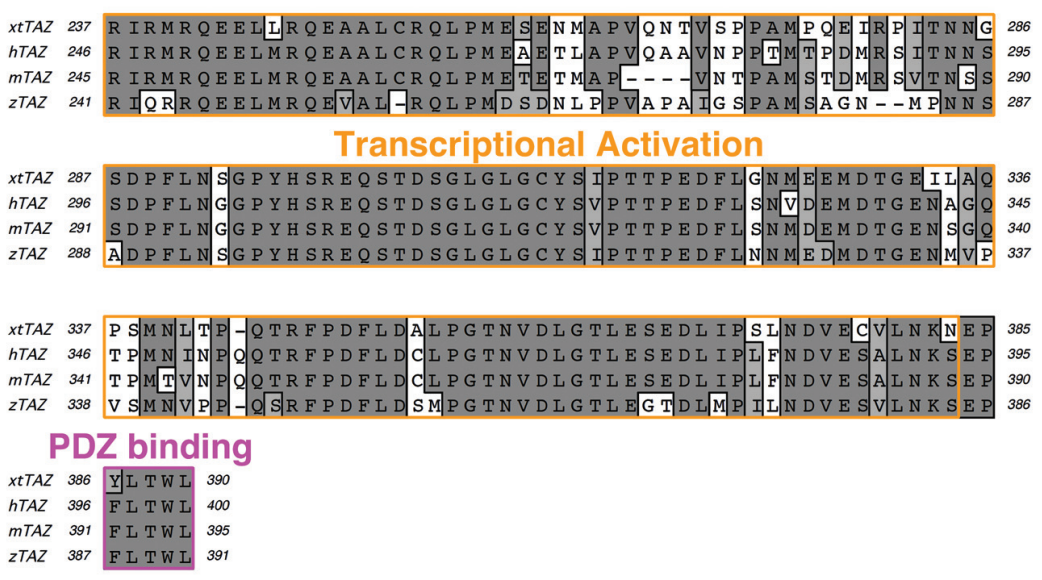

Fig. 2. Comparisons of the TAZ protein sequences of Xenopus tropicalis and other vertebrate TAZ. The scheme of $x t T A Z$ is shown on the top. The amino acid sequence of XTTAZ was aligned with those of human TAZ (hTAZ; accession no. NP_056287), mouse TAZ (mTAZ; NP_598545) and zebrafish TAZ (zTAZ; NP_001032785). The dark or light background highlights identical or similar residues, respectively. The domains of TAZ are boxed using the following colors and abbreviations; light-green, TB: TEAD binding domain; blue, 14-3-3: 14-3-3 binding motif; red, WW: WW domain; yellow, TA: transcriptional activation domain; pink, PDZ: PDZ binding motif. 
Method: Neighbor Joining; Best Tree; tie breaking = Systematic

Distance: Uncorrected ("p")

Gaps distributed proportionally

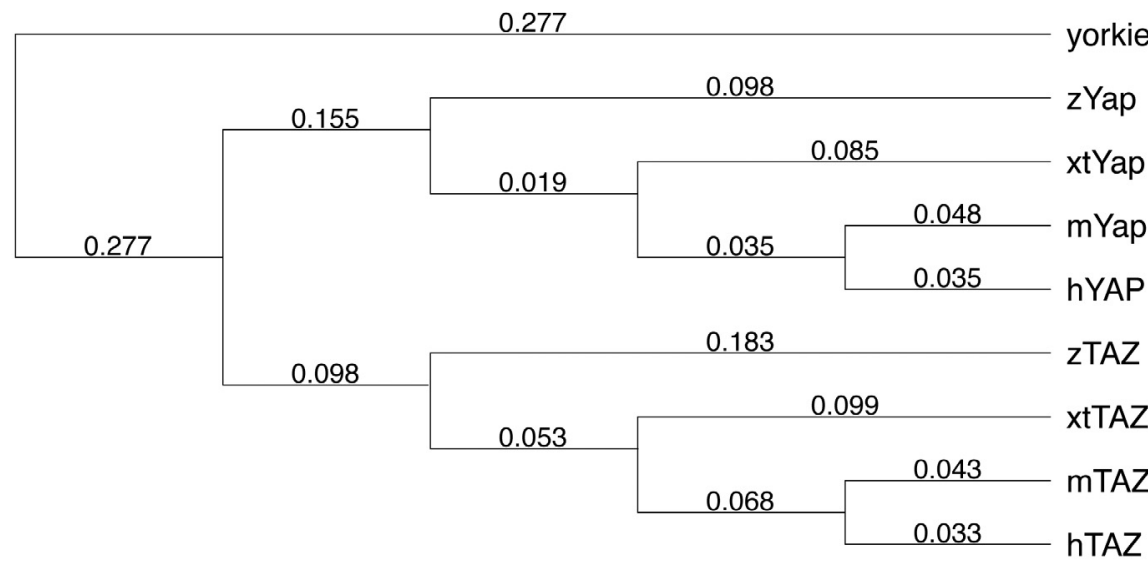

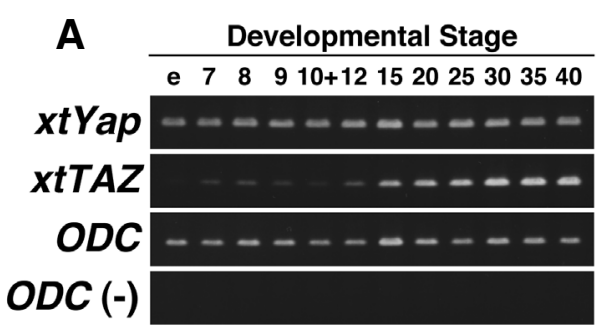

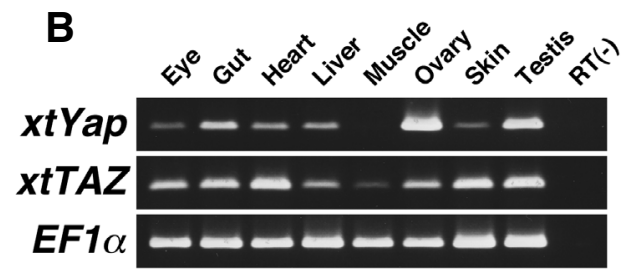

Fig. 3 (Left). Rooted phylogeny of the Yap, TAZ and Yorkie amino acid sequences. The following amino acid sequences were used in the analysis: human Yap (hYAP; accession no. NP_001123617); mouse Yap (mYap; NP_033560); Xenopus tropicalis Yap (xtYap; AB551789); zebrafish Yap (zYap; NP_001132952); human TAZ (hTAZ; NP_056287); mouse TAZ (mTAZ; NP_598545); Xenopus tropicalis TAZ (xtTAZ; AB551790); and zebrafish TAZ (zTAZ; NP_001032785); Yorkie (NP_001036568).

Fig. 4 (Right). Comparison of the $x t$ Yap and $x t T A Z$ expression patterns. (A) Temporal expression profiles of xtYap and xtTAZ. RNA samples were extracted from embryos at various developmental stages, as indicated above each lane. e, Unfertilized egg. ODC (-) is a negative control for ODC. (B) Expression profiles of xtYap and xtTAZ in adult Xenopus tissues. RNA samples were extracted from Xenopus tissues, as indicated above each lane. $R T(-), R T-P C R$ without reverse transcriptase in the testis cDNA synthesis reaction.

presomitic mesoderm from the neurula stage to the tailbud stage. We also show that $x t T A Z$ is expressed in the facial connective tissues, the midbrain-hindbrain boundary, the hindbrain, the branchial arch, and in the trunk neural crest cells at the tadpole stage. Furthermore, we demonstrate that $x t T A Z$ is expressed in the presomitic mesoderm and hypaxial myoblasts, but not in the differentiated somite. These observations suggest that $x t Y a p$ and $x t T A Z$ are expressed widely, and that in some tissues (e.g., the center of the neural plate and cranial neural crest cells for $x t Y a p$, and the hindbrain and presomitic mesoderm and hypaxial myoblasts for $x t T A Z$ ), their expression levels are high. It has been reported that the large amounts of Yap and TAZ produced by overexpression cannot be inhibited by the endogenous Hippo signaling in Drosophila and mammalian cells (Dong et al., 2007). There is no information as to when Hippo signaling is active during Xenopus development. The region highly expressing Yap or TAZ may be resistant to be the Hippo signaling.

Yap and TAZ have been shown to function as transcriptional
Fig. 5. Spatial expression pattern of $x t$ Yap during the early development of Xenopus tropicalis. (A-K) Spatial expression of $\mathrm{xtYap}$ was analyzed by whole-mount in situ hybridization. Anterior is at the left of each panel. (A) Unfertilized egg, (B) 8-cell stage, (C) early gastrula, (G) tailbud stage, (H) late tailbud stage, and (I) tadpole stage in lateral view. (D) Late gastrula, (E) neurula stage, and (F) late neurula stage in dorsal view. (J) Transverse section of (E). (K) Transverse section of (G). The line position is indicated in $\mathbf{( E , G ) . ~ S c a l e ~ b a r s , ~} 50$ $\mu \mathrm{m}$. Abbreviations: ba, branchialarch; ca, cloaca; cn, center of neural plate; $f_{C}$, facial connective tissues; $h g$, hindgut; mh, midbrain-hindbrain boundary;
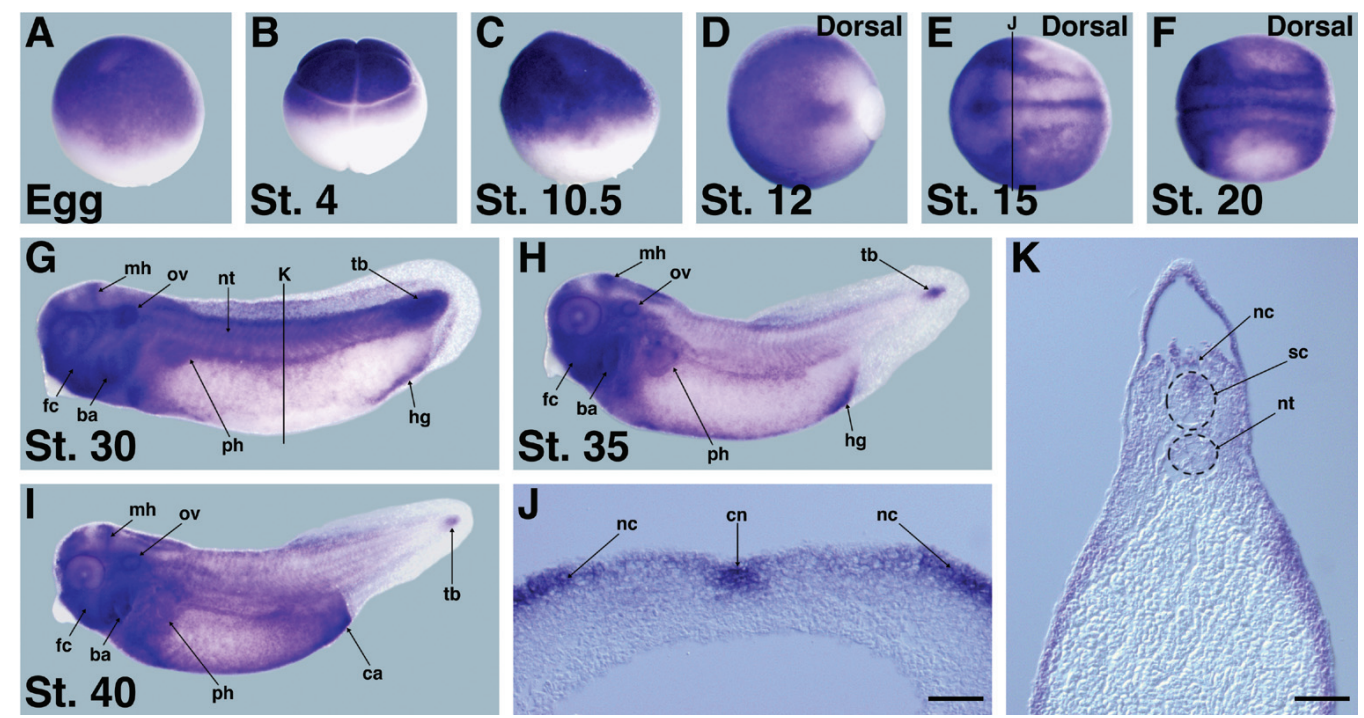

; nc, neural crest; nt, notochord; ov, otic vesicle; ph, pronephros; sc, spinal cord; tb, tailbud. 

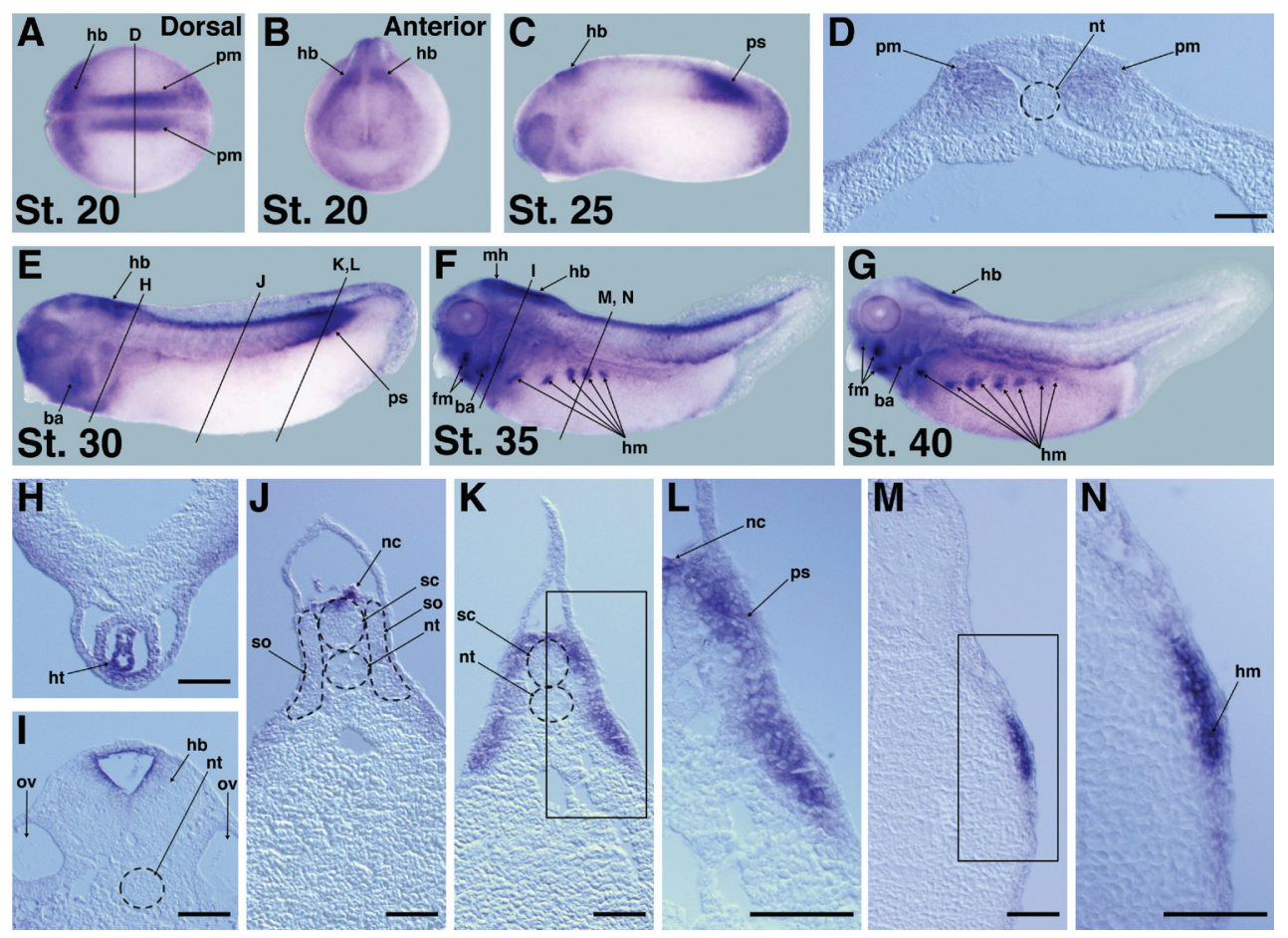

Fig. 6. Spatial expression pattern of $x t T A Z$ during the early development of Xenopus tropicalis. (A-N) Spatial expression of xtTAZ was analyzed by whole-mount in situ hybridization. Late neurula stage in (A) dorsal view and (B) anterior view, (C) early tailbud stage, (E) tailbud stage, (F) late tailbud stage, and $(\mathbf{G})$ tadpole stage in lateral view. (D) Transverse section of (A). (H, J, K) Transverse section of (E). (I, M) Transverse section of $(\mathbf{F})$. The line position is indicated in (E-G). (L) Higher magnification of (K). (N) Higher magnification of (M). The box position is indicated in (K,M). Scale bars, $50 \mu \mathrm{m}$. Abbreviations: ba, branchial arch; $f m$, facial musculature; $h b$, hindbrain; $h t$, heart; $h m$, hypaxial myoblasts; mh, midbrain-hindbrain boundary; nt, notochord; pm, paraxial mesoderm; ps, presomitic mesoderm; sc, spinal cord; so, somite. coactivators for Tead, Tbx5, and Pax3. Tead family members are major transcriptional factors that interact with Yap and TAZ and induce cell proliferation and epithelial-mesenchymal transition (Vassive et al., 2001, Mahoney et al., 2005, Wang et al., 2009). The mammalian Tead family has four members, i.e., Tead1-4 (NTEF-1, ETEF-1, DTEF-1 and RTEF-1, respectively). However, inÄXenopus only two Tead genes have been identified: $x$ Tead 1 (xNTEF-1) and $x$ Tead3 ( $x$ DTEF-1); a previous study has characterized the expression patterns of $x$ Tead1 and $x$ Tead3 (Naye et al., 2007). Some of the expression domains of $x t Y a p$ and $x t T A Z$ overlap with those of $x$ Tead1 and $x$ Tead3. The $x$ Tead1 and $x$ Tead3 transcripts were predominantly detected in the eyes, embryonic brain, somites, and heart. Therefore, xtYap and TAZ can act as binding partners for xTead1 and xTead3 in these tissues. In other regions, in which $x$ tYap or $x t T A Z$ but not the Tead genes are expressed, these coactivators may function together with other transcriptional factors. For example, in the present study, we show that $x t T A Z$ is expressed at the edge of the migrating hypaxial muscle. Pax3 is expressed in Xenopus hypaxial muscle at the same stage, and functions in abdominal muscle formation in the mouse (Martin and Harland, 2001; Tajbakhsh et al., 1997; Tremblay et al., 1998). It has been reported that the transcriptional activity of $\mathrm{Pax} 3$ is coactivated by TAZ (Murakami et al., 2006). Therefore, TAZ may act as a coactivator of $\mathrm{Pax} 3$ at the edges of proliferating and migratory hypaxial muscles to cancel signaling by the Hippo pathway.

\section{Materials and Methods}

\section{Plasmid construction}

Using the BLAST search algorithm (Altschul et al., 1990), we identified from JGI Xenopus tropicalis v4. 1 the genomic clones that correspond to Xenopus tropicalis Yap and TAZ. The full-length ORFs of $x t Y a p$ and $x t T A Z$ were amplified by PCR from the corresponding cDNAs of Xenopus tropicalis embryos and adult testes, respectively. The following primer sets (forward and reverse, respectively) were used:

xtYap 5'-ATGGAGCCCGGATCCCAG-3'

5'-CTATAACCACGTGAGGAAACTTTC-3';

$x t T A Z, \quad 5 '$-ATGAATCCGAGCCCACATCTCC-3' and

5'-CTACAGCCAGGTGAGGTAGGGCTC-3'. Each clone was subcloned into the pGEM-T-easy vector, and $x t Y a p$ was subcloned into the pCS2p (+) vector. pCS2p- $x$ tYap and pGEM-xtTAZ were used for synthesis of the in situ hybridization probes.

\section{Alignment of sequences and construction of a phylogenic tree}

Multiple protein sequence alignments and the generation of a phylogenic tree were performed using the CLUSTALW tool of the MacVector software (MacVector Inc.). The following sequences were used: hYAP (accession no. NP_001123617); mYap (NP_033560); zYap (NP_001132952); hTAZ (NP_056287); mTAZ (NP_598545); zTAZ (NP_001032785); and Yorkie (NP_001036568).

\section{Semi-quantitative RT-PCR}

Total RNA samples were isolated from various developmental stage embryos and adult tissues using RNAiso (Takara Bio Inc.), and $2 \mu \mathrm{g}$ of total RNA was used as the template for first-strand cDNA synthesis using SuperScript II Reverse Transcriptase according to the manufacturer's instructions (Invitrogen). The cDNA (1 $\mu \mathrm{l})$ was used as a template in the $\mathrm{PCR}$. The following primer sets (forward and reverse, respectively) were used:

xtYap 5'-TCACTCCAGAGACGAAAGCACTGAC-3'

5'-CGTTGAGGATGTCGGAACTGAGAG-3';

xtTAZ 5'-GGCATTCACACATACGGGACAGAG-3'

5'-GCAGGGCTGGAAGTTATTTGTTGG-3';

ornithine decarboxylase $(O D C)$ and elongation factor $1 \alpha(E F 1 \alpha)$ were used as internal controls. The primer sets for ODC and EF1 $\alpha$ were described in Sekizaki et al. (2004).

\section{Whole-mount in situ hybridization and histology of embryos}

Xenopus tropicalis embryos were obtained by artificial fertilization according to the method of Showell and Conlon (2009) with modifications, 
cultured in $10 \%$ Steinberg's solution, and staged according to the scheme of Nieuwkoop and Faber (1994). Whole-mount in situ hybridization analysis was performed as described previously (Harland, 1991). Stained embryos were re-fixed in Bouin's solution overnight, dehydrated, embedded in paraffin, and sectioned at a thickness of $10 \mu \mathrm{m}$.

\section{Acknowledgements}

We thank Dr David L. Turner for the generous gift of pCS2 vector. We also thank Eri Torikai for her assistance. Xenopus tropicalis was provided by the National Bio-Resource Project (NBRP) of the MEXT, Japan. This work was supported by a Grant-in-Aid for Young Scientists (to S.T.) from the Japan Society for the Promotion of Science.

\section{References}

ALTSCHUL, S.F., GISH, W., MILLER, W., MYERS, E.W. and LIPMAN, D.J. (1990). Basic local alignment search tool. J Mol Biol 215: 403-410.

BADOUEL, C., GARG, A. and MCNEILL, H. (2009). Herding Hippos: regulating growth in flies and man. Curr Opin Cell Biol. 21: 837-43.

DI PALMA, T., D'ANDREA, B., LIGUORI, G.L., LIGUORO, A., DE CRISTOFARO, T., DEL PRETE, D., PAPPALARDO, A., MASCIA, A. and ZANNINI, M. (2009). TAZ is a coactivator for Pax8 and TTF-1, two transcription factors involved in thyroid differentiation. Exp Cell Res 315: 162-175.

DONG, J., FELDMANN, G., HUANG, J., WU, S., ZHANG, N., COMERFORD, S.A., GAYYED, M.F., ANDERS, R.A., MAITRA, A. and PAN, D. (2007). Elucidation of a universal size-control mechanism in Drosophila and mammals. Cell 130: 1120-1133.

HARLAND, R.M. (1991). In situ hybridization: an improved whole-mount method for Xenopus embryos. Methods Cell Biol 36: 685-695.

HONG, J.H., HWANG, E.S., MCMANUS, M.T., AMSTERDAM, A., TIAN, Y., KALMUKOVA, R., MUELLER, E., BENJAMIN, T., SPIEGELMAN, B.M., SHARP, P.A. et al. (2005). TAZ, a transcriptional modulator of mesenchymal stem cell differentiation. Science 309: 1074-1078.

HOSSAIN, Z., ALI, S.M., KO, H.L., XU, J., NG, C.P., GUO, K., QI, Z., PONNIAH, S., HONG, W. and HUNZIKER, W. (2007). Glomerulocystic kidney disease in mice with a targeted inactivation of Wwtr1. Proc Natl Acad Sci USA 104: 1631-1636.

JIANG, Q., LIU, D., GONG, Y., WANG, Y., SUN, S., GUI, Y. and SONG, H. (2009). yap is required for the development of brain, eyes, and neural crest in zebrafish. Biochem Biophys Res Commun 384: 114-119.

LEI, Q.Y., ZHANG, H., ZHAO, B., ZHA, Z.Y., BAI, F., PEI, X.H., ZHAO, S., XIONG Y. and GUAN, K.L. (2008). TAZ promotes cell proliferation and epithelialmesenchymal transition and is inhibited by the hippo pathway. Mol Cell Biol 28: 2426-2436.

MAHONEY, W.M., JR., HONG, J.H., YAFFE, M.B. and FARRANCE, I.K. (2005). The transcriptional co-activator TAZ interacts differentially with transcriptional enhancer factor-1 (TEF-1) family members. Biochem J 388: 217-225.

MAKITA, R., UCHIJIMA, Y., NISHIYAMA, K., AMANO, T., CHEN, Q., TAKEUCHI, T., MITANI, A., NAGASE, T., YATOMI, Y., ABURATANI, H. et al. (2008). Multiple renal cysts, urinary concentration defects, and pulmonary emphysematous changes in mice lacking TAZ. Am J Physiol Renal Physiol 294: F542-553.

MARTIN, B.L. and HARLAND, R.M. (2001). Hypaxial muscle migration during primary myogenesis in Xenopus laevis. Dev Biol 239: 270-280.

MORIN-KENSICKI, E.M., BOONE, B.N., HOWELL, M., STONEBRAKER, J.R., TEED, J., ALB, J.G., MAGNUSON, T.R., O'NEAL, W. and MILGRAM, S.L. (2006). Defects in yolk sac vasculogenesis, chorioallantoic fusion, and embryonic axis elongation in mice with targeted disruption of Yap65. Mol Cell Biol26: 77-87.

MURAKAMI, M., TOMINAGA, J., MAKITA, R., UCHIJIMA, Y., KURIHARA, Y., NAKAGAWA, O., ASANO, T. and KURIHARA, H. (2006). Transcriptiona activity of Pax3 is co-activated by TAZ. Biochem Biophys Res Commun 339: 533-539.

NAYE, F., TREGUER, K., SOULET, F., FAUCHEUX, C., FEDOU, S., THEZE, N and THIEBAUD, P. (2007). Differential expression of two TEF-1 (TEAD) genes during Xenopus laevis development and in response to inducing factors. Int $J$ Dev Biol 51: 745-752.

NIEUWKOOP, P.D. and FABER, J. (1994). Normal Table of Xenopus laevis Garland Publishing, New York.

NISHIOKA, N., INOUE, K., ADACHI, K., KIYONARI, H., OTA, M., RALSTON, A. YABUTA, N., HIRAHARA, S., STEPHENSON, R.O., OGONUKI, N. et al. (2009). The Hippo signaling pathway components Lats and Yap pattern Tead4 activity to distinguish mouse trophectoderm from inner cell mass. Dev Cell 16 398-410.

OVERHOLTZER, M., ZHANG, J., SMOLEN, G.A., MUIR, B., LI, W., SGROI, D.C. DENG, C.X., BRUGGE, J.S. and HABER, D.A. (2006). Transforming properties of YAP, a candidate oncogene on the chromosome 11q22 amplicon. Proc Nat Acad Sci USA 103: 12405-12410.

SEKIZAKI, H., TAKAHASHI, S., TANEGASHIMA, K., ONUMA, Y., HARAMOTO, Y. and ASASHIMA, M. (2004). Tracing of Xenopus tropicalis germ plasm and presumptive primordial germ cells with the Xenopus tropicalis DAZ-like gene. Dev Dyn 229: 367-372.

SHOWELL, C. and CONLON, F.L. (2009). Egg collection and in vitro fertilization of the western clawed frog Xenopus tropicalis. CSH Protoc 2009: pdb prot5293.

SUDOL, M. (1994). Yes-associated protein (YAP65) is a proline-rich phosphoprotein that binds to the $\mathrm{SH} 3$ domain of the Yes proto-oncogene product. Oncogene 9: 2145-2152

TAJBAKHSH, S., ROCANCOURT, D., COSSU, G. and BUCKINGHAM, M. (1997). Redefining the genetic hierarchies controlling skeletal myogenesis: Pax-3 and Myf-5 act upstream of MyoD. Cell 89: 127-138.

TIAN, Y., KOLB, R., HONG, J.H., CARROLL, J., LI, D., YOU, J., BRONSON, R., YAFFE, M.B., ZHOU, J. and BENJAMIN, T. (2007). TAZ promotes PC2 degradation through a SCFbeta-Trcp E3 ligase complex. Mol Cell Biol27: 6383 6395.

TREMBLAY, P., DIETRICH, S., MERICSKAY, M., SCHUBERT, F.R., LI, Z. and PAULIN, D. (1998). A crucial role for Pax3 in the development of the hypaxia musculature and the long-range migration of muscle precursors. Dev Biol 203 : 49-61.

VASSILEV, A., KANEKO, K.J., SHU, H., ZHAO, Y. and DEPAMPHILIS, M.L. (2001). TEAD/TEF transcription factors utilize the activation domain of YAP65, a Src/Yes-associated protein localized in the cytoplasm. Genes Dev 15: 12291241.

WANG, K., DEGERNY, C., XU, M. and YANG, X.J. (2009). YAP, TAZ, and Yorkie: a conserved family of signal-responsive transcriptional coregulators in animal development and human disease. Biochem Cell Biol 87: 77-91. 


\section{Further Related Reading, published previously in the Int. J. Dev. Biol.}

See our recent Special Issue Placenta edited by Joan S. Hunt and Kent L. Thornburg at: http://www.ijdb.ehu.es/web/contents.php?vol=54\&issue=2-3

A conserved MRF4 promoter drives transgenic expression in Xenopus embryonic somites and adult muscle Timothy J. Hinterberger

Int. J. Dev. Biol. (2010) 54: 617-625

Zygotic VegT is required for Xenopus paraxial mesoderm formation and is regulated by Nodal signaling and Eomesodermin Masakazu Fukuda, Shuji Takahashi, Yoshikazu Haramoto, Yasuko Onuma, Yeon-Jin Kim, Chang-Yeol Yeo, Shoichi Ishiura and Makoto Asashima

Int. J. Dev. Biol. (2010) 54: 81-92

Dynamic expression pattern of distinct genes in the presomitic and somitic mesoderm during Xenopus development Audrey Bourdelas, Hong-Yan Li, Clémence Carron and De-Li Shi

Int. J. Dev. Biol. (2009) 53: 1075-1079

Differential expression of two TEF-1 (TEAD) genes during Xenopus laevis development and in response to inducing factors François Naye, Karine Tréguer, Fabienne Soulet, Corinne Faucheux, Sandrine Fédou, Nadine Thézé and Pierre Thiébaud Int. J. Dev. Biol. (2007) 51: 745-752

Myoskeletin, a factor related to Myocardin, is expressed in somites and required for hypaxial muscle formation in Xenopus Hui Zhao, Martha L. Rebbert and Igor B. Dawid

Int. J. Dev. Biol. (2007) 51: 315-320

The mob as tumor suppressor (mats1) gene is required for growth control in developing zebrafish embryos Yuan Yuan, Shuo Lin, Zuoyan Zhu, Wenxia Zhang and Zhi-Chun Lai Int. J. Dev. Biol. (2009) 53: 525-533.
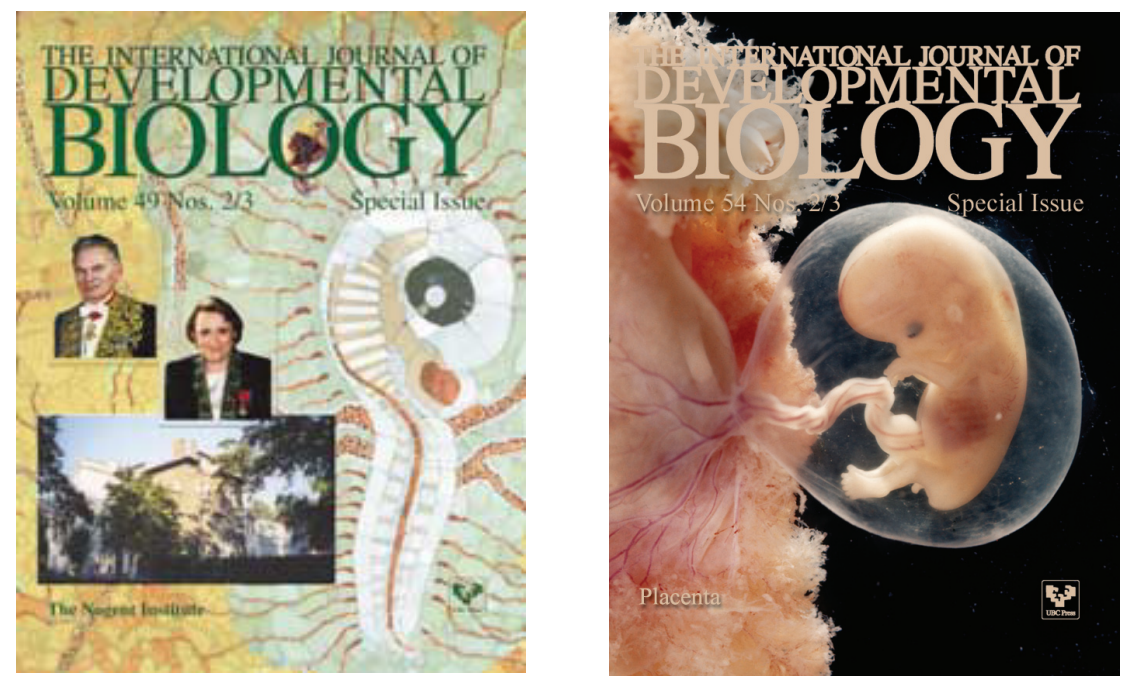

5 yr ISI Impact Factor $(2009)=3.253$

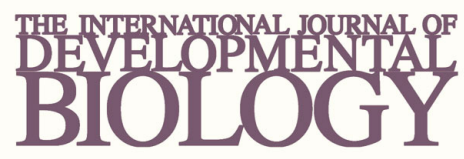

Volume 54 Nos. 6/7

Special Issue

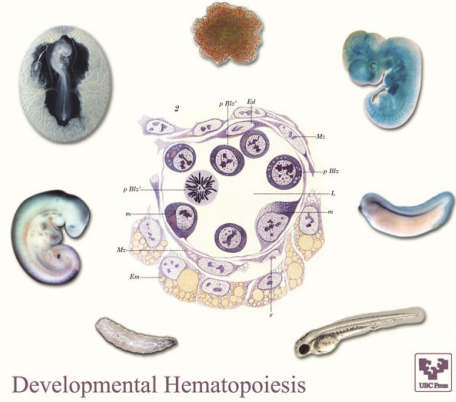

